Price Continuity Rules And Insider Trading

By Prajit K. Dutta, Columbia University And Ananth Madhavan, University of Pennsylvania

\author{
Discussion Paper No.661 \\ Current Version: May 1993 \\ Original Version: February 1991
}

$$
d p-9293-661
$$




\title{
Price Continuity Rules and Insider Trading
}

\begin{abstract}
This paper analyzes the impact on price dynamics of price continuity-depth requirements that restrict transaction-to-transaction price changes when some traders possess private information about asset values. Price continuity rules enable insiders to slowly exploit their information over time. Paradoxically, more stringent price continuity requirements may actually improve market efficiency indirectly by increasing insider profits and inducing more traders to become informed at cost. We also demonstrate that the autocorrelation in returns induced by price-continuity rules provides a rationale for the use of technical trading rules by outsiders who effectively 'free-ride' off the private information of insiders. We show that price continuity requirements can make both insiders and outsiders better off by reducing the rents to market makers.
\end{abstract}




\title{
Price Continuity Rules and Insider Trading
}

\author{
Prajit K. Dutta \\ and \\ Ananth Madhavan* \\ Current Version: May, 1993 \\ Original Version: February, 1991
}

- Department of Economics. Columbia University, New York. NY 10027 and the Wharton School of the University of Pennsvlvania. Philadelphia. PA 19104. respectively. We have benefitted from the helpful comments of Jeffrey Banks. Andrew Caplin. Gary Gorton. Bruce Grundy, Rangarajan Sundaram. and seminar participants at the University of Rochester and Johns Hopkins University. Research support from the Columbia University Council for Social Science Research (Dutta) and the Research Foundation of the University of Pennsylvania and GeewaxTerker Research Fund (Madhavan) is gratefully acknowledged. Any errors are entirely our own. 


\section{Introduction}

In many markets. the prices set by market makers must satisfy price continuity-depth requirements. Yet, these rules have largely been ignored in both theoretical and empirical analyses of market microstructure. ${ }^{1}$ This paper examines the effects of price continuity requirements on price formation and market efficiency.

The simplest price continuity rules place upper limits on the absolute change in price during trading hours. ${ }^{2}$ More sophisticated rules link the allowable change in price to order flow. For example, New York Stock Exchange (NYSE) Rule 104 requires the specialist to maintain a 'fair and orderiy market.' To achieve this. the NYSE provides price continuitydepth guidelines to its specialists that vary across stocks and are determined by the price range and normal trading volume of the stock. For instance. a stock trading between $\$ 20$ and $\$ 29 \frac{7}{8}$ with average daily share volume in the previous month (excluding trades of 25,000 or more) of $10.000-24,999$, the maximum price change permissible for volume of 3,000 shares is $\$ \frac{7}{8}$. The NYSE reports that in $1988,92.1 \%$ of all transactions of 1,000 shares or less traded with a price change of 0 or $\frac{1}{8}$ from the immediately preceding trade. Adherence to these guidelines is one criterion by which specialists are evaluated, and accordingly specialists who perform poorly according to these measures risk having their stocks reassigned to others or not being assigned more profitable stocks in the future. Usually there are special provisions for suspending continuity requirements after a public announcement. On the NYSE for example, the specialist can call a trading halt (with the permission of a floor official) in the event of an impending public announcement.

Price continuity rules are usually justified as a way to maintain price stability for 'small' investors. However, there may be a trade-off between price stability and market efficiency. In an early analysis of the problem, Black (1971) notes:

There is a right amount of price continuity for every stock under any given

'Black (1971) was the first to discuss the effect of price continuity requirements. Hamao and Hasbrouck (1992) examine empirically the effect of continuity rules on the Tokyo Stock Exchange.

${ }^{2}$ In futures markets, these limits are intended to act as circuit breakers in the event of an extremely unusual event such as a crash. 
set of market conditions, and either more or less than that is undesirable. Large changes in price caused by the arrival of new information affecting the value of the stock are desirable. Large changes in price that are caused by a temporary imbalance between supply and demand are undesirable.

Black's comments suggest the existence of an 'optimal' level of exchange-mandated price continuity. In this paper, we examine whether there exists an economic rationale for price continuity rules, and their implications for transaction prices.

We consider the following model: A group of traders (or insiders) possess private information concerning the full-information value of a security. Insiders and uninformed traders without private information trade in the market until a random date at which time the full-information price is publicly revealed. In each period, a market maker who acts as a trader of last resort determines the asset's price. subject to price continuity requirements of the types discussed above. ${ }^{3}$ In this paper, we analyze the effect on price formation and market efficiency of continuity-depth requirements that restrict price movements when some traders possess private information.

Since informed traders are always on one side of the market, either buying or selling, their trading creates price trends when price movements are limited by continuity requirements. This prediction is consistent with empirical evidence that successive transactions on the NYSE tend to occur on the same side of the quote. We also analyze the impact on price formation and efficiency of changes in price continuity rules. In equilibrium. if the number of insiders is fixed, price continuity requirements do not affect the rate of convergence of prices. As a result, if private information can be acquired at cost. more stringent price continuity requirements actually increase market efficiency by increasing the rewards to becoming informed. However, this leads to the duplication of investment in the production of short-term information that will soon become public. imposing social costs.

\footnotetext{
${ }^{3}$ In existing models of trading under asymmetric information. e.g., Kyle (1985), prices are not restricted.
} 
Finally, we analyze the optimal price continuity rule under alternative criteria for an exchange. We demonstrate an important rationale for the existence of price continuity rules; restrictions on price movements limit the market power of market makers who may otherwise move prices significantly in response to order flow, thereby narrowing the effective bid-ask spread and increasing the welfare of all investors.

The rest of the paper proceeds as follows: In Section 2 we set up the model, and describe the strategic choices of the players. Section 3 analyzes the strategies of a monopolistic insider, and in Section 4, we analyze the multiple insider case. The analysis is extended in Section 5 to the case where traders can acquire information at cost. In Section $i$, we discuss the determination of an optimal price continuity rule. Finally, Section 8 summarizes the paper and offers suggestions for further research. Proofs are in the appendix.

\section{The Model}

\subsection{The Structure of Trading}

Consider the market for a single risky asset that can be traded at dates $t=1,2 \ldots, \tilde{\tau}$. The security is a claim to the cash flows from a project which begins to vield revenue or dividends at a random date $\tilde{\tau}$ in the future. The dividend paid at time $t(t>\tau)$ is denoted by $\check{d}_{t}$. We assume $\check{d}_{t}$ is independently drawn from a distribution with unknown mean $d>0$. The event date $\tau$ is an integer-valued random variable: on this date the mean of the dividend process $d$ is publicly announced and the first (random) dividend payment is made. The announcement is made just before trading in that period begins. As an aside. date $\tau$ can be interpreted as the time when news of an impending news event first becomes public (as opposed to the event day itself), allowing the market maker to halt trading according to exchange rules. We assume that the probability that the event occurs in the next period. given no announcements to date. is $\phi \in(0.1)$. In other words. the time to revelation is geometrically distributed, and is thus independent of the elapsed time. 
With these assumptions, the expected value of the security is simply the expected present value of the stochastic stream of dividends. Let $r>0$ denote the risk-free rate of interest, and denote by $v$ the expected value of future dividends given private information about $d$. Hence, the probability that the event occurs $t$ periods from now is $(1-\phi)^{t-1} \phi$. The expected present discounted value of the dividend stream starting $t$ periods from now is $\frac{d}{r} \frac{1}{(1+r)^{r}}$. Consequently, the expected value of the asset in any period prior to the announcement date is a constant equal to:

$$
v=\sum_{t=1}^{\infty} \frac{(1-\phi)^{t-1} \phi}{(1+r)^{t}}\left(\frac{d}{r}\right)=\frac{\phi}{\phi+r}\left(\frac{d}{r}\right) .
$$

We turn to a formal description of the objectives and information of market participants. The stochastic structure of the economy is common knowledge. The focus of the paper is on the behavior of two group of traders. The first group is referred to as insiders or informed traders and they obtain private information regarding the mean of the dividend process, $d$, at time 0 . Insiders are assumed to be risk-neutral. In particular, we are interested in the question of how insiders profitably exploit their information in the preevent epoch and the effect of their strategies on price dynamics. There are $N \geq 1$ insiders, indexed by $i=1, \ldots, N$. Let $q_{i t}$ represent the order quantity of informed trader $i$ with the convention that $q>0$ denotes a trader purchase and $q<0$ a trader sale.

In addition to traders with private information, other agents with no private information. referred to as outsiders or uninformed traders, also trade. These traders are liquidity-motivated and their trade, $\tilde{x}_{t}$, is a random variable that is independent across time-periods with mean zero and variance $\sigma_{x}^{2}$, where $x>0(x<0)$ denotes net purchase (sale) as before.of trading, such as Kyle (1985) or Admati and Pfleiderer (1988), serving to camouflage the actions of insiders.

Denote by $p_{t}$ the security's price at time $t$. The price is determined by a single market maker or specialist on the basis of the net order flow in period $t$, denoted by $Q_{t}=\sum_{i}^{N} q_{i t}+x_{t}$. Only market orders can be submitted and the specialist is required to fill all orders. 


\subsection{Price Continuity Requirements}

We assume that the market maker is subject to a price continuity-depth requirement of the form $\left|p_{t}-p_{t-1}\right| \leq \lambda Q_{t}$. This rule states that the maximum permissable price revision is linearly related to the net order imbalance facing the specialist in period $t$, and is consistent with the types of continuity rules in use in equity markets. The coefficient $\lambda>0$ summarizes the liquidity of the market, with lower values of $\lambda$ implying more shares can be traded for a given price change. We define market depth as $\lambda^{-1}$, the minimum order flow associated with a unit price change, a definition analogous to that in Kyle (1985). In Section $j$ we analyze an alternative price continuity rule where the price adjustments are linked to past and current volume and show that our results extend to this more complicated case. ${ }^{4}$ When the dividend announcement is made at time $\tau$, we assume prices adjust to the new level implied by the release of dividend information, i.e., the market maker calls a trading halt and revises prices while the continuity rules are suspended.

To focus attention on the effect of the price continuity rule imposed upon the market maker, we assume that the requirement is always binding, i.e., the market maker sets the highest (lowest) permissable price when faced with a net buy (sell) order imbalance. This assumption is consistent with a market maker who maximizes expected profits in each period. Accordingly, the price revision rule can be written as a linear function. $p_{t}=p_{t-1}+\lambda Q_{t}$. Such price behavior could also be viewed, following Hakansson, Beja, and Kale (1985), as a representation of the price adjustment process in an automated trading system that does not require dealers.

A linear price adjustment rule also arises in Kyle (1985). However, in our model. the market depth is determined by the exchanges whereas in Kyle's model. it originates endogenously from the learning process of an uninformed market maker. For appropriate values of $\lambda$, our model yields the Kyle model. Our focus, however. is on the effects of

\footnotetext{
${ }^{t}$ The case of price limits independent of volume are not analyzed here. Such price limits are primarily designed to act as circuit breakers in the event of an exceptional event. such as the October 1987 crash. and not to maintain short- run price stability.
} 
binding price continuity requirements on price formation.

\subsection{Trader's Strategies}

Having described the market environment. we turn now to a description of the strategic behavior of informed traders. Suppose there are $N$ informed traders. At time $t$, trader $i(i=1, \ldots N)$ submits an order $q_{i t}$ based on all available information including the previous trading history generated by past prices and quantities and the anticipation of the price (which we write as $p_{t}\left(Q_{t}\right)$ ) set by the specialist given the strategies of the other traders. A strategy for an informed trader is a decision rule to select the order quantity $q_{i t}$ as a function of the particular history observed. The decision rules are said to be Markov (or history independent) if they depend only on the beginning period price, $p_{t-1}$.

A vector of strategies forms a Nash equilibrium if each player's strategy maximizes discounted profits evaluated at date 0 , conditional upon the strategies of all other players being correctly conjectured. A vector of strategies form a sub-game perfect equilibrium if, after each history, the continuation strategies. form a Nash equilibrium in the remaining game. A sub- game perfect equilibrium is a Markov perfect equilibrium if the decision rules are Markov. In this paper we consider subgame perfect equilibria in the trading game.

\section{The Monopolist Insider}

To develop the intuition for our results, we consider first the case where there is a single insider. The optimization problem faced by a monopolist insider is:

$$
\max _{\left\{q_{t}\right\}} E\left[\sum_{t=0}^{\tilde{T}} \frac{\left(v-\tilde{p}_{t}\right) q_{t}}{(1+r)^{t}}\right]
$$

where $\tilde{p}_{t}=p_{t-1}+\lambda q_{t}+\lambda x_{t}$ with probability $1-\dot{\phi}$ and $v$ with probability $\phi$. The expectation in (1) is taken over $\tilde{\tau}$ and $\tilde{x}_{t}{ }^{5}$ After $\tilde{\tau}$. the security pays a liquidating dividend and all trading ceases.

\footnotetext{
${ }^{5}$ The initial price $p_{0}$ is the unconditional expectation of the value of the security at time 0 .
} 
Observe that the price continuity requirement on price adjustment implies the insider's trading problem is intertemporal in nature. If the security is undervalued initially, insider purchases lead to price increases, lowering potential profits in the future. We will show that the optimal dynamic strategy is to trade a fixed proportion of the deviation between the market price and the value of the asset.

Let $V\left(p_{t-1}\right)$ denote the value function corresponding to the optimization problem (1) for any time $t<\tau$. The associated optimality equation is (using the Bellman principle):

$$
V\left(p_{t-1}\right)=\max _{q_{t}} E\left[\left(v-p_{t-1}-\lambda q_{t}-\lambda x_{t}\right) q_{t}+\beta V\left(p_{t-1}+\lambda q_{t}+\lambda x_{t}\right)\right],
$$

where $\beta \equiv \frac{1-\phi}{1+r}$ is the effective discount factor. Note, that although the pre-event trading period lasts (with probability 1) a finite number of periods. the appropriate horizon for the insider's decision problem is infinite. This is because at every date in the pre-event period, there is a positive probability that the insider's information will remain private for another period.

It will be analytically convenient to consider as a basic state variable the deviation of price from true value rather than the price itself. Accordingly, define $z_{t-1} \equiv v-p_{t-1}$ as the state variable and denote by $W$ the associated value function. Of course, $W\left(z_{t-1}\right)=$ $r\left(v-z_{t-1}\right)$.

Proposition 1 For $t<\tau$, the optimal trading strategy of the monopolist insider is a linear function of the price deviation:

$$
q_{t+1}^{*}\left(z_{t}\right)=\hat{\theta} z_{t}
$$

where $\hat{\theta}$ is inversely proportional to $\lambda$. Further, the value function is:

$$
W\left(z_{t}\right)=\left(\frac{1-\lambda \hat{\theta}}{2 \lambda}\right) z_{t}^{2}+\gamma(\beta) \lambda^{*} .
$$

i.e., discounted profits are a quadratic function of the initial price deviation. Further. expected prices converge geometrically to the true value of the asset:

$$
E[z t]=(\alpha(1-0))^{t} z_{t}
$$


where $\alpha=\alpha(\beta) \in(0,1)$ and $\gamma(\beta)$ are constants described in the appendix. In particular. $\alpha$ is independent of the continuity-depth parameter $\lambda$.

Remarks: The parameter $\alpha$ is a metric for market efficiency since it is the rate at which prices converge to the full information value in the pre-event period. Prices jump to the present value of future dividends following the earnings announcement. ${ }^{6}$ Lower values of $\alpha$ are associated with greater market efficiency, i.e., faster rates of convergence. Proposition 1 shows that the continuity-depth parameter $\lambda$ does not effect the rate of convergence of prices. $\alpha$.

Proposition 1 forms an important benchmark case where the insider is a monopolist. The trader s strategy is such that the rate of price convergence is independent of market depth. The effect on insider's profits of market depth is determined by the amount of initial mispricing $z_{0}$. If this is high, more stringent price continuity rules allow an insider to extract larger rents by trading bigger volume (although price dynamics are unchanged in $\lambda$ ). If $z_{0}$ is small, and an insider does not wish to trade much, more stringent price continuity rules have the adverse effect of making prices insensitive to liquidity trading. So, for large mispricing, the effect of more stringent price continuity rules (i.e., lower $\lambda$ ) is not to slow the rate at which private information is impounded in prices. but to increase the informational rents of the insider.

\section{Multiple Insiders}

In this section, we are concerned with the analysis of price dynamics resulting from competition among insiders and later on competition with outsiders. ${ }^{7}$ Purely for notational ease in the immediate sequel we discuss the case where $N=2$. The generalization of our resuits to $N>2$ is straightforward (and is presented at the end of the proof of Proposition

\footnotetext{
"Meulbroek (1991) examines the stock price run-up before takeovers and finds price paths suggestive of the slow exploitation of information by insiders. In particular, she finds that 43 percent of the run-up in the 20 days before the announcement occurs on days when insiders traded.

'See also Holden and Subrahmanyam (1992), who analyze the effect of competition among insiders in a dynamic model. In their model, market makers are not constrained by price continuity rules. The focus of our analysis is on the consequences of such rules on price formation.
} 
2). Again for notational ease we ignore strategic uninformed traders in the present section. and focus exclusively on the competition between informed traders. in the presence of liquidity motivated noise traders.

Trader $i$ s best response problem. given the trading strategy of trader $j$, denoted by $q_{j}(p)$, is:

$$
\max _{\left\{q_{i t}\right\}} E\left[\sum_{t=0}^{\bar{\tau}} \frac{\left(v-p_{t}\right) q_{i t}}{(1+r)^{t}}\right],
$$

where $p_{t}=p_{t-1}+\lambda\left(q_{i t}+q_{j t}\left(p_{t-1}\right)\right)+\lambda x_{t}$. As in the monopolist insider case, it is convenient to think of trader $i$ as the residual trader who picks the deviation of price from fundamentals in the next period. Let $z_{t-1}=v-p_{t-1}$ be the current price deviation. Then. writing $W_{i}$ for the value function for trader $i$. some algebra yields the following Bellman equation ${ }^{8}$ for the trader:

$$
W_{i}\left(z_{t-1}\right)=\max _{z_{t}} E\left[\frac{z_{t}\left(z_{t-1}-z_{t}-\lambda q_{j}\left(z_{t-1}\right)-\lambda x_{t}\right)}{\lambda}+\beta W_{i}\left(z_{t}\right)\right],
$$

where $\beta=(1-\theta) /(1+r)$ and $z_{t}$ is the next period's price deviation. Suppose now that trader $j$ s strategy is a linear function of the price deviation:

$$
q_{j}\left(z_{t}\right)=\theta_{j} z_{t}
$$

where $\theta_{j}>0$. Such a strategy is motivated by the linearity of the monopolist insider's optimal trading strategy. We explore two elements of the analysis: the optimal trading strategy or best response of insider $i$ as a residual trader and. secondly, the existence and character of equilibria in linear strategies.

The following resuit establishes that there exists a $\theta^{*} \in\left(0, \frac{1}{2 \lambda}\right)$ such that if $\theta_{j}=\theta^{*}$. then the best response of trader $i$ is a linear trading rule with $\theta_{i}=\theta^{*}$. In this equilibrium. prices converge geometrically to $v$ at a rate faster than the monopolist insider case.

Proposition 2 There is a proportional trading rule $\theta^{*} z$ for the insiders, which constitutes a Markov-perfect equilibrium given the linear adjustment rule $p_{t}=p_{t-1}+\lambda Q_{t}$ for the

\footnotetext{
${ }^{\circ}$ The value function $W_{i}$ will depend on trader $j$ 's trading strategy $q_{j}$, and should therefore be written as $W_{i}\left(\cdot ; q_{j}\right)$. However, in the immediate sequel we will construct a particular form for $q_{j}$ and the value function will refer to this strategy.
} 
specialist. The expected rate of price convergence is geometric:

$$
E\left[z_{t}\right]=\left(\alpha_{2}(1-0)\right)^{t} z_{0}
$$

where $\alpha_{2} \in(0,1)$ is a constant. Further, $\alpha_{2}<\alpha$. i.e.. the rate of convergence of prices is faster with 2 traders than with a monopolist insider.

Proposition 2 describes the unique symmetric equilibrium when traders adopt linear Markov strategies. Expected discounted profits are given by:

$$
\begin{aligned}
W_{i}\left(z_{t}\right) & =\frac{\left(1-\lambda \theta^{*}\right)\left(1-2 \lambda \theta^{*}\right)}{\lambda}\left[z_{t}^{2}+\frac{\beta}{1-\beta} \frac{\sigma_{x}^{2}}{2} \lambda^{2}\right] . \\
& \equiv \frac{k}{2} z^{2}+\ell .
\end{aligned}
$$

We discuss the relationship between the continuity-depth parameter $\lambda$ and the rate of price convergence later.

\section{Continuity Requirements and Market Efficiency}

We now investigate the variation in equilibrium behavior as the market primitives, the number of informed traders $N$ and the market liquidity $\lambda$, change. We examine the effect of varying $(N, \lambda)$ on price efficiency and the size of informational rents.

Recall that the (symmetric) equilibrium strategies are given by: each informed trader

trades $\theta(N, \lambda)\left[v-p_{t-1}\right]$ at price $p_{t-1}$ and discounted expected profits are $\frac{k(N, \lambda)}{2}\left(v-p_{0}\right)^{2}+$ $\ell(N, \lambda)$ if the initial price is $p_{0}$. Moreover, in the rounds before disclosure, prices converge geometrically at the rate $1-N \lambda \theta(N, \lambda) \equiv \alpha(N, \lambda)$. Proposition 3 examines the effect of changes in $N$ and $\lambda$ on market efficiency and profits.

Proposition 3 In a symmetric Markov equilibrium:

1. The rate of price convergence $\alpha$ is independent of market liquidity $\lambda$.

2. However. $\alpha$ is a declining function of the number of insiders $N$. 
3. Insiders' profits $W\left(z_{0} ; N, \lambda\right)$ are decreasing (increasing) in $\lambda$ if the initial price deviation $z_{0}$ is greater (less) than $z(\lambda)$.

4. The value function $W\left(z_{0} ; N, \lambda\right)$ is decreasing in $N$ for every initial price deviation $z_{0}=v-p_{0}$.

5. The total losses of market makers is decreasing in $N$.

The proposition shows that for a fixed number of informed traders, price efficiency, as measured by $\alpha$, is independent of the continuity-depth parameter $\lambda$. As expected, prices are more efficient with a greater number of informed traders. An immediate corollary of proposition 3 is that the trading proportion $\theta(N, \lambda)$ is inversely proportional to $\lambda$, i.e.. given a more continuous price regime. in equilibrium all traders exactly offset the potential increase in smoothing by larger volume of transactions. The intuition underlying the effect on total profits of a change in the price continuity rule $\lambda$, is similar to the monopoly case. Consider a lowering of $\lambda$. This relieves price pressure consequent upon any insider trade, provided the insiders do want to trade at current prices. i.e., provided $z$ is large. If $z$ is small, a lower $\lambda$ means that liquidity trading is less likely to make $z$ larger; this affects insider $\pi$ adversely. On the other hand an increase in the number of informed traders reduces informational rents.

In our analysis so far. we have assumed that some traders are exogenously endowed with private information. Suppose now that traders can endogenously determine whether or not to become informed at cost. We assume that there is a pre-game round in which the number of insiders is determined endogenously. The payoffs to this game are determined by insider profits in the subsequent trading rounds, which depends on the total number of traders who choose to become informed. The Nash equilibrium for the pre-game determines the number of insiders.

Formally, suppose at time 0 , a trader can become informed at cost $c>0$. If the current price is $p$ and the number of other informed traders is $N-1$, then the expected 
returns to becoming informed is:

$$
\begin{aligned}
R_{N}(p) & =\int_{0}^{\infty} W(v-p ; N, \lambda) d f(v)-c \\
& =\frac{k(N, \lambda)}{2} \int_{0}^{\infty}(v-p)^{2} d f(v)+\ell(N, \lambda)-c
\end{aligned}
$$

where $f$ is the prior density of $\tilde{v}$ given public information.

Indeed, the total profits can written, using the results in the appendix, as:

$$
W(z ; N, \lambda)=g(N)\left[\frac{z^{2}}{\lambda}+\frac{\beta}{1-\beta} \frac{\sigma_{x}^{2}}{2} \lambda\right],
$$

where $g(N)$ is known from Proposition 3 to be a decreasing function. Writing $\psi(p) \equiv$ $\int_{0}^{\infty}(v-p)^{2} d f(v)$, the number of traders who choose to acquire information is given implicitly by

$$
g\left(N^{*}\right)=\frac{c}{\frac{\psi(p)}{\lambda}+\frac{3}{1-B} \frac{\sigma_{x}^{2}}{2} \lambda} .
$$

Clearly the larger the expected deviation of current price from the true value, the greater the number of informed traders. The number of insiders will also depend on the continuitydepth parameter $\lambda$, as shown by the following proposition:

Proposition 4 If traders can acquire information at cost. stricter price continuity-depth requirements (i.e.. lower values of $\lambda$ ) are associated with faster price convergence (i.e.. with lower values of $\alpha$ ), if and only if $\frac{2 \psi(p)}{\lambda^{2}} \geq \frac{\beta}{1-3} \frac{\sigma_{x}^{2}}{2}$.

Proposition 4 implies that more stringent price continuity rules can. in some circumstances, improve efficiency. At first glance, this result appears counter-intuitive. The intuition, however, is straightforward: Lower values of $\lambda$ increase the expected profits of insiders. inducing a higher $N^{*}$. and implying faster price convergence. From a policy viewpoint this is not necessarily the best way to increase market efficiency, however, since it induces a larger number of socially wasteful searches.

Since variations of the price continuity rule studied in this paper are also observed in security markets. it is worth exploring the robustness of our results to such variations. 
In particular, the form of the NYSE guidelines for transaction-to-transaction price movements suggest a price continuity rule which restricts the price changes in the $t$-th round to some moving average of volume in current and immediately preceding trading rounds. Consider a rule such as:

$$
p_{t}=p_{t-1}+\dot{\lambda}\left[\frac{1}{2} Q_{t-1}+\frac{1}{2} Q_{t}\right],
$$

where price change is limited by the average volume in the current and preceding round. Clearly, (12) is equivalent to (the notationally simpler) price revision rule $p_{t}=p_{t-1}+$ $\lambda\left[Q_{t-1}+Q_{t}\right]$, where $\lambda=\frac{1}{2} \hat{\lambda}$. In the rest of this section we will demonstrate that our conclusions are qualitatively unaffected by this variation. In particular. we will analyze in detail the (notationally simpler) case of a monopolistic insider and show that the conclusions remain unchanged. The reader can verify that the same is true for the multiple insiders case.

The following proposition characterizes the consequent price dynamics and the insider's profits.

Proposition 5 For $t<\tau$, the optimal trading strategy of the monopolist insider is a linear function of the price deviation and the aggregate quantity traded in the previous round:

$$
q_{t}^{*}\left(z_{t-1}\right)=\theta_{1} z_{t-1}-\left(1-\theta_{2}\right) Q_{t-1}
$$

where $\theta_{1}$ is inversely proportional to $\lambda$. whereas $\theta_{2}$ is independent of $\lambda$. Further, the value function is:

$$
W\left(z_{t-1}\right)=a \lambda+b\left(\frac{z_{t-1}^{2}}{\lambda}+\lambda Q_{t-1}^{2}\right)+c z_{t-1} Q_{t-1}
$$

where $a . b, c$ are constants independent of $\lambda$, i.e., discounted profits are a quadratic function of the initial price deviaion and volume. Further, expected prices converge to the true value of the asset at a rate which is independent of the continuity-depth parameter $\lambda$.

The proposition shows that our analysis can be extended straightforwardly to more complicated price continuity rules based on moving averages of volume as opposed to current 
volume alone. Our basic conclusions in this more complex case are unaltered, so from this point onward we will assume the continuity rule is a function of current volume alone.

\section{The Optimal Price Continuity Rule}

In this section, we derive the optimal price continuity rule within the linear class under a set of alternative criteria. In doing so, we provide an economic rationale for the existence of such rules. ${ }^{9}$ For simplicity, we restrict our attention to the base case where the number of informed traders is exogenous and there are no strategic outsiders. ${ }^{10}$ We begin with a set of possible selection criteria for the exchange, within the class of linear rules:

(C1) Minimize excess volatility subject to non-negative expected discounted profits for the specialist.

(C2) Maximize the combined expected profits of insiders and outside investors subject to non-negative expected discounted profits for the specialist.

(C3) Maximize market liquidity (depth) subject to non-negative expected discounted profits for the specialist.

(C4) Allow the specialist to select the continuity parameter $\lambda$ subject to excess price variability being bounded by a constant, $L$.

Criterion (C1) is usually cited as the main reason for maintaining continuity rules. The term 'excess volatility' requires further explanation. Price movements reflect two components: a permanent component warranted by fundamentals, and a transitory component induced by noise trading. Attempting to minimize the former component only reduces market efficiency and is not a viable alternative in the long run. (Actually, both short- and long-run objectives yield the same rule, but minimizing short-run temporary price shocks is a more natural goal.) The other selection criteria are self-explanatory, and coincide with the stated objectives of exchanges. While criteria (C1)-(C3) impose restrictions on the specialist directly, $(\mathrm{C} 4)$ allows the specialist to choose the linear rule

\footnotetext{
We thank the referee for suggesting this to us.

${ }^{10}$ It is straightforward to extend our results to these more general cases: our qualitative results are unchanged.
} 
subject to a constraint on price variability. Another reasonable criterion is to maximize price efficiency subject to constraints on specialist profits. This objective is not included because the rate of price convergence in our model is independent of the choice of the continuity rule. The following proposition establishes that there exists an unique price continuity rule satisfying all four selection criteria.

Proposition 6 There exists an unique linear price continuity rule satisfying criteria (C1)-(C3), and an upper bound $L^{*}$ such that this rule will be selected by the specialist in accordance with $(C 4)$. Further, for $L>L^{*}$, the expected profits of the specialist are strictly positive and increase with $L$.

Remarks: Proposition 8 has two important implications: First. there exists a continuity rule consistent with all four exchange objectives. Continuity rules can increase liquidity, improve price stability in the short-run, and maximize the net gains of all traders. Second. continuity rules limit the expected profits of the specialist. Transitory order imbalances originating from liquidity traders can cause price movements attributable to 'bid-ask bounce.' Price continuity requirements are usually justified as a method of reducing price volatility that is not warranted by changes in fundamentals. However, without restrictions of some sort on permissible price movements, a non-competitive market maker would set raise (lower) prices when faced with excess demand (supply), thereby widening the effective bid-ask spread and price variability. Thus, proposition 8 provides a rationale for the existence of continuity rules in a market where dealers possess market power.

In the appendix, we prove that the optimal continuity-depth parameter is inversely related to the standard deviation of noise trading, i.e., to $\sigma_{x}$. Intuitively, if the expected nlume of noise trading increases. maintaining price stability requires a less sensitive price adjustment rule. Similarly, with greater noise trading, a smaller continuity parameter is required to maintain a given level of specialist expected profits. This discussion suggests that the optimal price continuity-depth parameter vary by security, with thickly traded securities having more stringent continuity requirements. 


\section{Conclusions}

In many security markets. there are institutional barriers to price movements, but there has been little analysis of the effect of such rules on price formation. This paper analyzes the impact on price dynamics of price continuity-depth requirements that restrict price changes when some traders possess private information about asset values. Price continuity rules enable insiders to slowly exploit their information over time. We show that irrespective of the number of insiders, the rate of convergence of prices to the fullinformation value is independent of the price continuity requirements since insiders adjust their rate of trading according to the continuity requirements. Paradoxically, more stringent price continuity requirements may actually improve market efficiency indirectly by increasing insider profits and inducing more traders to become informed at cost.

We analyze the exchange's choice of a price continuity rule under alternative selection criteria. We demonstrate an important rationale for the existence of price continuity rules; restrictions on price movements limit the market power of market makers who may otherwise move prices significantly in response to order flow. There exists an optimal continuity rule that maximizes the total expected profits of traders and also minimizes transaction-to-transaction price movements subject to a non- negativity constraint on market maker expected profits. The optimal continuity rule is shown to vary systematically with the expected volume of liquidity trading, with active securities subject to more stringent requirements than thinly traded securities.

Finally, the analysis raises some interesting theoretical and empirical issues that lie beyond the scope of this paper. First, the model suggests a positive relation between the continuity parameter and market maker profits, given the expected volume of trading. This relation could be tested using intraday data on market maker positions such as those used by Hasbrouck and Sofianos (1992). Second. the model implies that the expected price change. conditional upon current information. is positively related to the current price change. This relation could also be tested on a transaction basis using intraday data. but requires some care in that the effect of continuity rules may be obscured by 
other microstructure effects such as inventory control by market makers, non-trading, or bid-ask bounce. Indeed. recent studies of intraday returns (e.g., Hasbrouck (1991)) suggest significant departures from the martingale property implicit in standard asymmetric information models. A cleaner test may be obtained by focusing on intraday returns prior to trading suspensions. Third. the use of technical trading strategies by outsiders may induce other forms of technical analysis. For example, suppose that no traders obtain private information in the pre-event period. Under the technical strategy analyzed above. a price increase triggered by noise traders leads to buying and hence future price increases, as technical traders chase a false trend. As this event occurs with positive probability, there is a potential profit opportunity for technical traders who pursue counter-cyclical trading strategies, i.e.. selling (buying) following a price increase (decrease). These traders. who act as market makers by supplying liquidity, earn positive expected profits in periods when there happen to be no informed traders buy systematically lose when some traders do in fact possess private information. Thus, the pre-event period may be characterized by unusually high volume. These are topics for future research. 


\section{References}

Admati, A. R.. and P. Pfleiderer, 1988, "A Theory of Intraday Trading Patterns," Review of Financial Studies. 1, 3-40.

Black. F.. 1971, "Toward a Fully Automated Stock Exchange: Part I." Financial Analysts Journal. 27, (July-August), 28-35, 44.

Foster, D., and S. Vishwanathan, 1990, "A Theory of Intraday Variations in Volume. Variance, and Trading Costs in Securities Markets," Review of Financial Studies. 3. 593-624.

Hakansson. N. A. Beja, and J. Kale, 1985. "On the Feasibility of Automated Market Making by a Programmed Specialist." Journal of Finance, 40. 1-20.

Hamao. Y.. and J. Hasbrouck, 1992, "Securities Trading in the Absence of Dealers: Trades and Quotes on the Tokyo Stock Exchange," Working paper, New York University.

Hasbrouck, J., 1991, "Measuring the Information Content of Stock Trades," Journal of Finance, 46, 178-208.

Hasbrouck. J., and G. Sofianos. 1992, "The Trades of Market-Makers: An Analysis of NYSE Specialists." Working paper. New York Stock Exchange.

Holden. C.. and A. Subrahmanyam. 1992. "Long-Lived Private Information and Imperfect Competition." Journal of Finance. 47, 247-270.

Kyvle, A.. 1985, "Continuous Auctions and Insider Trading," Econometrica, 53, 1315-1335.

Meulbroek, L. K., 1991, An Empirical Analysis of Insider Trading and the Stock Market. Securities and Exchange Commission. 


\section{Appendix}

\section{Proof of Proposition 1}

From the insider's perspective, the price schedule takes the form:

$$
p_{t}=p_{t-1}+\lambda q_{t}+\lambda x_{t}
$$

The optimization problem faced by a monopolist insider is:

$$
\max _{\left\{q_{t}\right\}} E\left[\sum_{t=0}^{\bar{\tau}} \frac{\left(v-\tilde{p}_{t}\right) q_{t}}{(1+r)^{t}}\right]
$$

where from equation (A.1), $\tilde{p}_{t}=p_{t-1}+\lambda q_{t}+\lambda x_{t}$ with probability $1-\phi$ and $v$ with probability $\phi$. Let $V\left(p_{t-1}\right)$ denote the value function corresponding to the optimization problem (A.2) for any time $t<\tau$. The Bellman equation yields:

$$
V\left(p_{t-1}\right)=\max _{q_{t}} E\left[\left(v-p_{t-1}-\lambda q_{t}-\lambda x_{t}\right) q_{t}+\beta V\left(p_{t-1}+\lambda q_{t}+\lambda x_{t}\right)\right],
$$

where $\beta \equiv \frac{1-\phi}{1+r}$. Rewriting (A.3) by using $z \equiv v-p_{t-1}$ and $W(z) \equiv V\left(p_{t-1}\right)$ we obtain:

$$
W(z)=\max _{q} E[(z-\lambda q-\lambda x) q+\beta W(z-\lambda q-\lambda x)] .
$$

Without loss of generality, suppose the deviation between $v$ and the previous price. $p_{t-1}$, is $z$ at the start of the period. Let $\tilde{y}=z-\lambda q-\lambda x$ be the deviation between value and price that results from a trade of size $q$ by the insider and $x$ by the noise traders. In other words, $y$ is the price deviation in the next period. and is a random variable because $x$ is stochastic.

The trader's choice problem then is equivalent to the choice of the expected deviation $z-\lambda q \equiv y$ conditional upon there being no information announcement. Equation (A.4) vields:

$$
W(z)=\max _{y} E\left[\tilde{y}\left(\frac{z-y}{\lambda}\right)+\beta W(\tilde{y})\right]
$$

which can be simplified to

$$
W(z)=\max _{y}\left[y\left(\frac{z-y}{\lambda}\right)+\beta E W(y-\lambda x)\right] .
$$


Note that in (A.5) the expectation is taken over $x$. Let the stationary optimal policy of the above problem be denoted by $h(z)$. This optimal choice satisfies (provided $W$ is differentiable):

$$
\frac{z-2 h(z)}{\lambda}+\beta E\left[W^{\prime}(\tilde{y})\right]=0 .
$$

The envelope theorem yields:

$$
W^{\prime}(z)=\frac{h(z)}{\lambda} .
$$

Consider solutions of the form $W(z)=\ell+\frac{k}{2} z^{2}$ and $h(z)=\alpha z$. Substituting these in (A.6)- (A.7) yields

$$
z(1-2 \alpha)+\beta k \alpha z=0
$$

which upon simplification leads to

$$
1-2 \alpha+\beta k \alpha=0
$$

Similarly from (A.7) we get:

$$
k=\frac{\alpha}{\lambda} \text {. }
$$

Equations (A.8) and (A.9) solve to yield optimal values of $k$ and $\alpha$. In particular, a little algebra reveals that $\alpha$ may be derived from the following quadratic function

$$
\beta \alpha^{2}-2 \alpha+1=0
$$

The quadratic has two roots. One root yields $\alpha>1$ and implies an expected price path which is explosive. This clearly cannot be part of an optimal trading strategy of the insider since it implies that he consistently buys overvalued stock and sells, in turn, undervalued stock. Therefore, the only meaningful solution to the quadratic is:

$$
\alpha=\frac{1-\sqrt{(1-\beta)}}{\beta}
$$

Substituting all of this back into the Bellman equation (A.5) we get

$$
\ell+\frac{\alpha}{2 \lambda} z^{2}=\frac{1}{\lambda} \alpha(1-\alpha) z^{2}+\beta \ell+\beta \frac{\alpha^{3}}{2 \lambda} z^{2}+\beta \frac{\alpha}{2 \lambda} \lambda^{2} \sigma_{x}^{2}
$$

which yields

$$
\ell=\frac{\beta}{1-3} \frac{\alpha}{2} \lambda \sigma_{x}^{2}=\frac{1-\sqrt{1-\beta}}{2(1-3)} \lambda \sigma_{x}^{2}
$$


From standard arguments we then know that as a solution to the optimality equation. $W$ is uniquely defined as the value function. The first order condition (A.6) is also a sufficient condition for optimality if the maximand is concave. The only term of any consequence in the right hand side of (A.5) is the coefficient of $y^{2}$, which is $\frac{\beta \alpha}{2}-1$, and this coefficient is negative so that the maximization problem is in fact strictly concave. Further $\alpha z$ is the unique trading strategy which maximizes a risk-neutral informed trader's expected profits when faced with a linear price adjustment schedule operated by the specialist. From the strict concavity of the objective in (A.5), the uniqueness of the optimal trading strategy follows.

Recall $\alpha=h(z) / z$ where $h(z)=z-\lambda q^{*}(z)$. Here $q^{*}(z)$ is the optimal trading volume at price deviation $z$. This implies that $\alpha=1-\lambda^{\frac{q^{*}(z)}{z}}$, so that:

$$
q^{*}(z)=\frac{(1-\alpha)}{\lambda} z \equiv \theta z
$$

where:

$$
\theta=\frac{\sqrt{(1-\beta)}-(1-\beta)}{\beta \lambda}
$$

\section{Proof of Proposition 2:}

We first characterize the best response of trader $i$ given that $j$ adopts a linear strategy. Then we discuss the equilibrium conditions. Rewriting (6) we obtain:

$$
W_{i}(z)=\max _{y}\left[\frac{y\left(\left(1-\lambda \theta_{j}\right) z-y\right)}{\lambda}+\beta E W_{i}(y-\lambda x)\right] .
$$

Denote the stationary policy for this problem by $h_{i}(z)$. Assuming for the moment that $W_{i}$ is in fact differentiable. the optimal choice must satisfy the following first-order and envelope conditions:

$$
\frac{\left(1-\lambda \theta_{j}\right) z-2 h(z)}{\lambda}+\beta E\left[W_{i}^{\prime}(h(z)-\lambda x)\right]=0,
$$

and

$$
W_{i}^{\prime}(z)=\frac{\left(1-\lambda \theta_{j}\right) h(z)}{\lambda}
$$

Consider a solution of the form. $W_{i}(z)=\frac{k}{2} z^{2}+\ell, h(z)=\alpha_{2} z$. Substituting these in (A.14)-(A.15) yields:

$$
\left(1-\lambda \theta_{j}\right) z-2 \alpha_{2} z+3 k \lambda \alpha_{2} z=0
$$


and

$$
k=\frac{\left(1-\lambda \theta_{j}\right)}{\lambda} \alpha_{2}
$$

Then (A.16) yields:

$$
\left(1-\lambda \theta_{j}\right)-2 \alpha_{2}+\beta\left(1-\lambda \theta_{j}\right) \alpha_{2}^{2}=0 .
$$

From (A.17) it follows that:

$$
\alpha_{2}=\frac{1 \pm \sqrt{1-\left(1-\lambda \theta_{j}\right)^{2} \beta}}{\beta\left(1-\lambda \theta_{j}\right)} .
$$

Equation (A.18) suggests the possibility of cyclical behavior (i.e., $\alpha_{2}<0$ ), stable monotone price convergence (i.e., $\alpha_{2} \in(0,1)$ ), or even unstable monotone explosive behavior i.e.. $\alpha_{2} \geq 1$ ). We analyze the cyclical and monotone explosive cases below and show in particular that such behavior cannot arise in equilibrium. We begin with the case: $1-\lambda \theta_{j} \in(0,1)$, i.e.. a situation in which. in the absence of any noise trade and trade by i. $\operatorname{sgn}\left(z_{t}\right)=\operatorname{sgn}\left(z_{t-1}\right)$.

Lemma 1 Suppose $\left(1-\lambda \theta_{j}\right)>0$. Then, under the best response of trader $i$,

1. If $E\left[z_{t+1}\right]>z_{t}$, then $E\left[z_{t+2}\right]>E\left[z_{t+1}\right]$. Conversely, if $E\left[z_{t+1}\right]<z_{t}$, then $E\left[z_{t+2}\right]<$ $E\left[z_{t+1}\right]$, i.e., the expected price deviation is either monotonically increasing or decreasing. Further, $\operatorname{sign}\left(z_{t}\right)=\operatorname{sign}\left(E\left[z_{t+1}\right\}\right)$.

2. The rate of convergence of prices is:

$$
\alpha_{2}=\frac{1-\sqrt{1-\left(1-\beta \lambda \theta_{j}\right)^{2}}}{\beta\left(1-\lambda \theta_{j}\right)}<\frac{1-\sqrt{1-3}}{\beta}=\alpha .
$$

3. The rate of adjustment $\alpha_{2}$ is decreasing in $\theta_{j}$.

\section{Proof of Lemma 1:}

Consider the Bellman equation (A.13). Denote the maximand by $U(z, y)$. We will show that $U$ is a strictly super-modular function. (A function $U(z, y)$ is super-modular if. for all $z^{\prime}>z, y^{\prime}>y, U\left(z^{\prime}, y^{\prime}\right)-U\left(z^{\prime}, y\right)>U\left(z, y^{\prime}\right)-U(z, y)$.) A sufficient condition is $U_{12}>0$. Note that $U_{12}=\frac{(1-\lambda \theta,)}{i}>0$. From strict super-modularity. a well-known 
implication is that the policy function $h$ is monotone. Then $E\left[z_{t+1}\right]=h\left(z_{t}\right)>z_{t}$ implies that $E\left[z_{t+2}\right]=h^{2}\left(z_{t}\right)>h\left(z_{t}\right)=E\left[z_{t+1}\right]$. Hence, $\alpha_{2}>0$. The converse is identical and this establishes (a) since $\alpha_{2}>0$.

To prove part (2), note that if $\alpha_{2}>1$, the implied expected price path is explosive. This implies strict losses for trader $i$, clearly a suboptimal strategy given the option to not trade at all. It is easy to check, by simple but tedious calculus that $\alpha_{2}<\alpha$, implying faster convergence. From the expression for $\alpha_{2}$, it is straightforward to check that $\alpha_{2}$ is a decreasing function of $\theta_{j}$, establishing part (3).

Remark: Recall that $\alpha_{2}=\frac{h(z)}{z}$. Since $h(z)=\left(1-\lambda \theta_{j}\right) z-\lambda q_{i}^{*}(z)$, we see that:

$$
q_{i}^{*}(z)=\frac{\left[\left(1-\lambda \theta_{j}\right)-\alpha_{2}\right] z}{\lambda} \equiv \theta_{i}\left(\theta_{j}\right) z
$$

i.e., demand is a linear function of the price deviation. To complete the argument we must show that there are linear strategies which are best responses to each other. Lemma 2 provides the basis for this result.

Lemma 2 Suppose that $1-\lambda \theta_{j}>0$. Then:

1. The best response of trader $i$ to trader $j$ 's strategy is:

$$
q_{i}^{*}(z)=\theta_{i}\left(\theta_{j}\right) z
$$

where:

$$
\theta_{i}\left(\theta_{j}\right)=\frac{\sqrt{1-\beta\left(1-\lambda \theta_{j}\right)^{2}}}{\beta\left(1-\lambda \theta_{j}\right) \lambda}\left(1-\sqrt{1-\beta\left(1-\lambda \theta_{j}\right)^{2}}\right) .
$$

2. The demand coefficient is strictly positive, i.e., $\theta_{i}\left(\theta_{j}\right)>0$.

3. The demand coefficient $\theta_{i}\left(\theta_{j}\right)$ is a continuous function of $\theta_{j}$, and satisfies the inequality $\left(1-\lambda \theta_{i}\left(\theta_{j}\right)\right)>0$.

4. The profits are a quadratic function of price deviation:

$$
W_{i}(z)=\frac{1-\sqrt{1-3\left(1-\lambda \theta_{j}\right)^{2}}}{2 \lambda \beta}\left[z^{2}+\frac{3}{1-\beta} \lambda^{2} \sigma_{x}^{2}\right] \text {. }
$$


Proof of Lemma 2: For (1), Equation (A.20) is immediate from the preceding discussion. Parts (2) and (3) are immediate. Note that $\alpha_{2}>0$ implies that $1-\lambda \theta_{i}\left(\theta_{j}\right)>0$. For part (4), the characterization of the value function follows from (A.15) and arguments identical to those in proposition 1.

To finish the proof, we now consider the case where $\left(1-\lambda \theta_{j}\right)<0$. In this case, the expected price deviation in the next period, $z\left(1-\lambda \theta_{j}\right)$, is the opposite sign of the initial deviation, and prices are cyclical or explosive. It is straightforward to demonstrate that such price paths cannot emerge in equilibrium. In the special case where $\left(1-\lambda \theta_{j}\right)=0$, prices converge in a single step. Then, the proposition follows directly from Lemmas 1 and 2 by applying the Brouwer fixed point argument to the space $\left[0, \frac{1}{\lambda}\right]$ and the function $\theta_{i}\left(\theta_{j}\right)$.

Remark: It is straightforward to check that the arguments for the two insider case generalize immediately to the $N$ insider problem. Hence, we only state the appropriate conclusion: there is a proportional trading rule $\theta^{*} z$ which constitutes a Markov perfect equilibrium; the expected rate of price converge is geometric at rate $\alpha_{N}$ and the profit of each insider, in equilibrium, is given by

$$
W_{i}\left(z_{t}\right)=\frac{\left(1-\lambda(N-1) \theta^{*}\right)\left(1-\lambda N \theta^{*}\right)}{2 \lambda}\left[z_{t}^{2}+\frac{\beta}{1-\beta} \lambda^{2} \sigma_{x}^{2}\right] .
$$

\section{Proof of Proposition ??}

Given that $\theta(N, \lambda)\left(v-p_{t-1}\right)$ is the trading strategy of the other $N-1$ traders, the $i$-the trader's maximization problem as a residual trader picking next period's price deviation $y$, given the current price deviation $z$, is

$$
\max _{y}\left[\frac{(1-(N-1) \lambda \theta) z-y}{\lambda} y+\beta E W(\tilde{y} ; N, \lambda)\right] .
$$

In (A.21) and henceforth in the proof we write $\theta$ instead of $\theta(N, \lambda)$. The first-order condition and envelope theorem condition are the exact analogues of (A.14) and (A.15) in the two insider case but we report them here for completeness:

$$
\begin{gathered}
\frac{(1-(N-1) \lambda \theta) z-2 y}{\lambda}+\beta E W^{\prime}(\tilde{y} ; N, \lambda)=0 \\
W^{\prime}(z)=\frac{1-(N-1) \lambda \theta}{\lambda} y .
\end{gathered}
$$


Substituting (A.23) in (A.22) and using the fact that $\theta z$ is a best response in the above problem (i.e., that $y=(1-N \lambda \theta) z$ ), we get:

$$
\beta(1-N \lambda \theta)^{3}+\beta \lambda \theta(1-N \lambda \theta)^{2}-(1-N \lambda \theta)+\lambda \theta=0 .
$$

Write $1-N \lambda \theta \equiv \alpha$, i.e., $\lambda \theta=\frac{1-\alpha}{N}$. Then,

$$
\beta(N-1) \alpha^{3}+\beta \alpha^{2}-(N+1) \alpha+1=0 .
$$

Denote the function on the left hand side of (A.25), $\zeta(N, \alpha)$. Elementary calculus yields $\zeta_{2}=3 \beta(N-1) \alpha^{2}+2 \beta \alpha-(N+1)$. It is easy to see that this quadratic can have at most one root in $(0,1)$, i.e. $\zeta(N, \cdot)$ has a single extremum in $\alpha \in(0,1)$. Further, $\zeta_{22}=6 \beta(N-1) \alpha+2 \beta>0$. i.e., the extremum is in fact a point at which $\zeta$ attains a minimum. Finally note that $\zeta(N, 0)=1$ and $\zeta(N, 1)=N(\beta-1)<0$. So there is exactly one $\alpha^{*}(N) \in(0,1)$ such that $\zeta\left(N, \alpha^{*}(N)\right)=0$.

We have proved 1. Notice further that $\zeta_{1}(N, \alpha)=\beta \alpha^{3}-\alpha<0$. Hence, $N^{\prime}>N$ implies that $\alpha^{*}\left(N^{\prime}\right)<\alpha^{*}(N)$. So 2. is proved.

From the value function computed above, we have

$$
W(z ; N, \lambda)=\alpha_{N}\left[\alpha_{N} \frac{N-1}{N}+\frac{1}{N}\right]\left[\frac{z^{2}}{\lambda}+\frac{\beta}{1-\beta} \frac{\sigma_{x}^{2}}{2} \lambda\right]
$$

By differentiating we get

$$
\operatorname{sgn} W_{\lambda}=\operatorname{sgn}\left[\frac{\beta}{1-\beta} \frac{\sigma_{x}^{2}}{2}-\frac{z^{2}}{\lambda^{2}}\right]
$$

3. immediately follows. Note that $\alpha_{N}<1$, and decreasing in $N$, implies that the convex combination $\alpha_{N} \frac{N-1}{N}+\frac{1}{N}$ is decreasing in $N$. From this 4. follows. Finally, 5. is proved by noting that increasing $N$ to $N+1$ leads to faster convergence than in the $N$ trader case, i.e., the aggregate informational rents are lower.

\section{Proof of Proposition 4}

We have seen that the number of insiders $N^{*}$ who choose to become informed is given by

$$
g\left(N^{*}\right)=\frac{c}{\frac{\psi(p)}{\lambda}+\frac{3}{1-\beta} \frac{\sigma_{2}^{2}}{2} \lambda}
$$


The proposition is proved by noting that $\frac{\psi(p)}{\lambda}+\frac{\beta}{1-\beta} \frac{\sigma_{2}^{2}}{2} \lambda$ is decreasing in $\lambda$ if and only if $\frac{2 \psi(p)}{\lambda^{2}} \geq \frac{3}{1-\beta} \frac{\sigma_{x}^{2}}{2}$.

\section{Proof of Proposition 5}

The price revision rule can be written as:

$$
z_{t}=z_{t-1}-\lambda\left(Q_{t-1}+q_{t}+x_{t}\right)
$$

In other words,

$$
\begin{aligned}
E\left[z_{t}\right] & =z_{t-1}-\lambda\left(Q_{t-1}+q_{t}\right) \\
E\left[Q_{t}\right] & =q_{t} .
\end{aligned}
$$

Denote. as before, $z \equiv z_{t-1}, y \equiv E z_{t}, x \equiv x_{t}$, and furthermore. $Q \equiv Q_{t-1}$. The Bellman equation is

$$
W(z, Q)=\max _{q} E[(z-\lambda Q-\lambda q-\lambda x) q+\beta W(z-\lambda Q-\lambda q-\lambda x, q+x)] .
$$

Again it will be more convenient to think of the insider as the residual trader who picks the expected price deviation $y$. Hence,

$$
W(z, Q)=\max _{y}\left[y\left(\frac{z-y-\lambda Q}{\lambda}\right)+\beta E W\left(y-\lambda x, \frac{z-y-\lambda Q}{\lambda}+x\right)\right] .
$$

We will investigate the following conjecture for the value function and optimal trading strategy respectively: $W(z, Q)=a_{0}+a_{1} z^{2}+a_{2} z Q+a_{3} Q^{2}$ and $q(z, Q)=\theta_{1} z-\left(1-\theta_{2}\right) Q$. Hence. $y=\left(1-\lambda \theta_{1}\right) z-\lambda \theta_{2} Q$. Substituting the conjecture, (A.30) can be rewritten as

$$
\begin{aligned}
W(z, Q)= & \max _{y}\left[y\left(\frac{z-y-\lambda Q}{\lambda}\right)+\beta a_{0}+\beta a_{1}\left(y^{2}+\lambda^{2} \sigma_{x}^{2}\right)\right. \\
& +\beta a_{2}\left(y \frac{z-y-\lambda Q}{\lambda}-\lambda \sigma_{x}^{2}\right) \\
& \left.+\beta a_{3}\left(\frac{(z-y)^{2}+\lambda^{2} Q^{2}-2(z-y) \lambda Q}{\lambda^{2}}+\sigma_{x}^{2}\right)\right] .
\end{aligned}
$$

The first-order condition from (A.31) yields, upon simplification:

$$
\left(1+\beta a_{2}\right)(z-2 y-\lambda Q)+2 \beta\left(\lambda a_{1} y-\frac{a_{3}}{\lambda}(z-y-\lambda Q)\right)=0 .
$$


Upon substituting the conjectured trading strategy we get:

$$
\begin{aligned}
\left(1+\beta a_{2}\right)\left(2 \lambda \theta_{1}-1\right)+2 \beta\left(\lambda a_{1}\left(1-\lambda \theta_{1}\right)-\theta_{1} a_{3}\right) & =0 \\
\left(1+\beta a_{2}\right)\left(2 \theta_{2}-1\right)+2 \beta\left(\left(1-\theta_{2}\right) a_{3}-\theta_{2} \lambda^{2} a_{1}\right) & =0 .
\end{aligned}
$$

Furthermore, (A.31) implies (by matching coefficients and then rearranging terms)

$$
\begin{aligned}
a_{0}(1-\beta)= & \beta \sigma_{x}^{2}\left(\lambda^{2} a_{1}-\lambda a_{2}+a_{3}\right) \\
a_{1}\left(1-\beta\left(1-\lambda \theta_{1}\right)^{2}\right)= & \theta_{1}\left(1-\lambda \theta_{1}\right)+\beta\left[a_{2} \theta_{1}\left(1-\lambda \theta_{1}\right)+a_{3} \theta_{1}^{2}\right] \\
a_{2}\left(\theta_{2}-2 \lambda \theta_{1} \theta_{2}+\lambda \theta_{1}-1\right)= & \left(1-\lambda \theta_{1}\right)\left(\theta_{2}-1\right) \\
& -\beta\left[2 a_{1}\left(1-\lambda \theta_{1}\right) \lambda \theta_{2}-a_{3}\left(\theta_{2}-2\right) \theta_{1}\right] \\
\frac{a_{3}}{\lambda^{2}}\left(1-\beta\left(1-\theta_{2}\right)^{2}\right)= & \left.\frac{\theta_{2}}{\lambda}\left(1-\theta_{2}\right)\right)+\beta\left[a_{2} \frac{\theta_{2}}{\lambda}\left(1-\theta_{2}\right)+a_{1} \theta_{2}^{2}\right] .
\end{aligned}
$$

Given the symmetry in the pairs of equations, (A.33)- (A.34) and (A.36)-(A.38), a natural conjecture is that $\lambda \theta_{1}=\theta_{2}$ and $a_{1} \lambda^{2}=a_{3}$. Substituting in (A.33) and simplifying, we get

$$
\left(2 \theta_{2}-1\right)\left[1+\beta a_{2}-\frac{2 \beta a_{3}}{\lambda}\right]=0
$$

Likewise (A.36) yields

$$
a_{3}\left[1-\beta\left(1-\theta_{2}\right)^{2}\right]=\theta_{2} \lambda\left(1-\theta_{2}\right)+\beta\left[a_{2} \theta_{2}\left(1-\theta_{2}\right) \lambda+a_{3} \theta_{2}^{2}\right] .
$$

In fact. suppose that $a_{3}=b \lambda$ (and hence, $a_{1}=\frac{b}{\lambda}$ ). Then. (A.39) yields $b=\frac{1+\beta a_{2}}{2 \beta}$. Upon substituting this in (A.40) we get

$$
\left(1+\beta a_{2}\right)\left[1-\beta\left(1-\theta_{2}\right)^{2}\right]=\left(1+\beta a_{2}\right) \beta\left(2-\theta_{2}^{2}\right)
$$

or

$$
1-3\left(1-\theta_{2}\right)^{2}=\beta\left(2-\theta_{2}^{2}\right)
$$

(A.41) can be seen to have a solution in $(0,1)$, for all $\beta>\frac{1}{3}$ (which we will assume is the case). By successive substitution the values of $a_{0}, b, a_{2}$, and $\theta_{1}$ can now be determined. The form of the optimal trading strategy, (13), and the discounted insider profits, (14), have therefore been established. 
To see that the price convergence is independent of $\lambda$, recall that $E\left[z_{t}\right]=\left(1-\lambda \theta_{1}\right) z_{t-1}-$ $\lambda \theta_{2} Q_{t-1}$. Since $\lambda \theta_{1}$ is independent of $\lambda$ (it is equal to $\theta_{2}$ which can be seen from (A.41) to be independent of $\lambda$ ) and since $Q_{0}=0$, it follow that $E\left[z_{1}\right]=\alpha z_{0}$ and $E\left[Q_{1}\right]=\frac{\theta_{2}}{\lambda} z$. In particular. $E\left[z_{1}\right]$ is independent of $\lambda$ and $E\left[Q_{1}\right]$ is inversely proportional to $\lambda$. By induction it follows that $E\left[z_{t}\right]$ is independent of $\lambda$ (and $E\left[Q_{t}\right]$ is inversely proportional to ג). The proposition is proved.

\section{Proof of Proposition 8}

First, consider the selection criterion (C1). As $p_{t}-p_{t-1}=\lambda\left(N \theta\left(v-p_{t-1}\right)+x_{t}\right)$, the excess transaction- to-transaction price variance, for a given fundamental value $z$, is $\sigma^{2}\left(p_{t}-p_{t-1}\right)=\lambda^{2} \sigma_{x}^{2}$. This variance represents the excess volatility in transactionto- transaction returns introduced by transitory demand shocks. as the demands of the informed traders are deterministic functions of $z$. Clearly, satisfying $(\mathrm{C} 1)$ implies choosing the smallest level of $\lambda$ consistent with non-negative specialist profits. (Note that this is the same action that would be chosen if the objective were to minimize the unconditional price variance, as the permanent price changes are not affected by continuity requirements over the long-run.)

Let $\Pi^{s}$ denote the expected trading profits of the specialist, i.e., $\Pi^{s} \equiv E\left[\left(p_{t}-v\right) Q_{t}\right]$. Then, from our previous results it follows immediately that:

$$
\Pi^{s}=E\left[\left(p_{t-1}+\lambda\left(N \theta\left(v-p_{t-1}\right)+x_{t}\right)-v\right)\left(N \theta\left(v-p_{t-1}+x_{t}\right)\right] .\right.
$$

Then:

$$
\Pi^{s}=E\left[\alpha N \theta\left(v-p_{t-1}\right)^{2}+\lambda x_{t}^{2}\right],
$$

where $\alpha=1-\lambda N \theta$ is a constant. From Proposition 3 , we know that $\alpha=\frac{1-\sqrt{1-\beta}}{\beta}$ is independent of $\lambda$ and that $N \theta=(1-\alpha) / \lambda$. Then, we can write the specialist's expected profits as a function of $\lambda$ :

$$
\Pi^{s}(\lambda)=-\alpha(1-\alpha) \sigma_{\eta}^{2} \lambda^{-1}+\lambda \sigma_{x}^{2}
$$

From equation (A.52), we see that $\Pi^{s}(\lambda)$ is strictly increasing in $\lambda$. Intuitively, higher values of $\lambda$ reduce the expected losses to informed traders and increase the expected gains from trading with uninformed traders. Clearly, the ex ante expected discounted profits 
of the specialist are proportional to the $\Pi^{s}$, where the proportionality constant depends only on the discount factor, $\beta$. Thus, setting $\Pi^{s}=0$, we see there exists a unique value of $\lambda$ given by $\lambda_{0}=\sqrt{\alpha(1-\alpha)} \sigma_{\eta} / \sigma_{x}$ satisfying (C1).

For (C2), we simply note that trading is a zero sum game, so that the expected profits of the informed and uninformed traders collectively are the expected losses of the market maker. Thus, setting the specialist's expected profits equal to zero is equivalent to satisfying criterion (C2). From equation (A.52), this yields the same value of $\lambda$ as criterion (C1). For (C3), we note that depth is just $\lambda^{-1}$, so by the earlier arguments, the same rule is chosen. Finally, for criterion (C4), we can choose a limit $L$ such that $L=\lambda_{0}^{2} \sigma_{x}^{2}$. As specialist profits are increasing in $\lambda$, the specialist chooses the same continuity rule as selected under $(\mathrm{C} 1)-(\mathrm{C} 3)$. 